\title{
PRODUCTIVITY, COST-BENEFIT ANAL YSES, MARKET PRICES AND CONSTRAINTS TO POULTRY FARMING IN THE NORTHERN DISTRICTS OF BANGLADESH
}

\author{
M. Saiful Islam*, Md. Ashraful Kabir ${ }^{1}$ and Ripon Kumar Dutta \\ Department of Zoology, University of Rajshahi, Rajshahi 6205, Bangladesh \\ ${ }^{1}$ Present address: Department of Biology, Holy Land College, Dinajpur 5200, Bangladesh \\ *Corresponding author: saifulzoo@yahoo.co.uk
}

\begin{abstract}
A total of 6,41,004 chickens were produced by 10 farms, of which Cobb 500 (broilers) in P1 were the highest in number (2,43,000) and G1 yielded the lowest (10,117 Fayoumi, RIR and Sonali breeds). The most common chicken breed reared in the area was Cobb 500 $(2,76,350)$ and the least common was ISA Brown $(43,163)$. With regard to productivity, significant differences existed among the farms $\left(\mathrm{F}_{9,103}=\right.$ 29.32; $\mathrm{P}<0.001)$, among the chicken breeds $\left(\mathrm{F}_{4,108}=3.03 ; \mathrm{P}<0.05\right)$, as well as between the government and private farms $\left(\mathrm{F}_{1,111}=6.17 ; \mathrm{P}<0.05\right)$. It was interesting to note that all the government farms were losing concerns $(\mathrm{G} 2>\mathrm{G} 3=\mathrm{G} 4>\mathrm{G} 1)$, while all the private farms were making profits $(\mathrm{P} 1>\mathrm{P} 3>\mathrm{P} 2>\mathrm{P} 6>\mathrm{P} 4=\mathrm{P} 5)$. The overall profitability index $(\mathrm{PI})$ values of government farms ranged between -0.09 and -0.13 , whereas those of private farms between 0.18 and 0.52 . Cobb 500 was the commonest but the least profitable breed, and Sonali was the most popular and cheapest one; and the growers achieved the maximum profit from Fayoumi. High feed cost, outbreak of diseases, inadequate supply of vaccines/medicines, lack of credit facilities and capital funding were recognized to be the major constraints to the poultry farming in the study area.
\end{abstract}

Key words: Productivity, poultry farms, constraints, market prices, profitability index.

সারাংশ: ১০টি খামারে সর্বমোট ৬,৪১,০০৪টি মুরগির মধ্যে কব ৫০০ (ব্রয়লার) জাতটি ছিল সবচেয়ে বেশি সংখ্যক (২,৪৩,০০০টি) যা ব্যক্তিগত খামার P1-এ উৎপাদিত এবং সবচেয়ে কম ছিল ফাউমি, আর.আই.আর. এবং সোনালী (১০,১১৭টি) যেগুলি সরকারি খামার G1-এ উৎপাদিত। অধ্যয়ন এলাকায় সবচেয়ে বেশি উৎপাদিত মুরগির জাতটি হচ্ছে

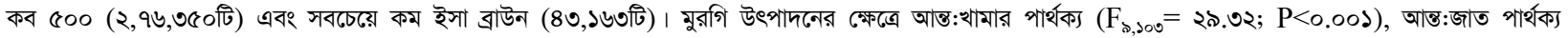
$\left(\mathrm{F}_{8, \text { ১০৮ }}=\right.$ ৩.০৩; $\mathrm{P}<0 . ০$ () এবং ব্যক্তিগত ও সরকারি খামারের মধ্যে পার্থক্য $\left(\mathrm{F}_{\text {১,১১১ }}=\right.$ ৬.১৭; $\left.\mathrm{P}<0 . ০ ৫\right)$ ছিল তাৎপর্যপূর্ণ। মজার ব্যাপারটি হলো সবগুলি সরকারি খামার লোকসান $(\mathrm{G} 2>\mathrm{G} 3=\mathrm{G} 4>\mathrm{G} 1)$ অথচ সবগুলি ব্যক্তিগত খামার লাভবান $(\mathrm{P} 1>\mathrm{P} 3>\mathrm{P} 2>\mathrm{P} 6>\mathrm{P} 4=\mathrm{P} 5)$ হিলেবে পরিচালিত হচ্ছিল। সার্বিক বিবেচনায় সরকারি খামারগুলিতে লাভের সূচক (profitability index, PI) -০.০৯ থেকে -০.১৩ এবং ব্যক্তিগত খামারগুলিতে তা' ০.১b- থেকে ০.৫২ নির্ণিত হয়। কব ৫০০ মুরগিটি সাধারণভাবে সব জায়গায় বেশি পাওয়া যায়, অন্য দিকে সোনালী জাতটি হচ্ছে জনপ্রিয় ও সস্তা এবং খামারিরা সবচেয়ে বেশি লাভবান হয় ফাউমি জাতের মুরগি পালন করে। খাদ্যের উচ্চ মূল্য, রোগের প্রাদুর্ভাব, টিকা/ওযুধের অপর্যাপ্ততা, কর্জ প্রাপ্তি ও মূলধনের অভাব জরীপ এলাকায় মুরগির খামারগুলির উন্নয়নে প্রধান অন্তরায় হিসেবে সনাক্ত করা হয়।

\section{Introduction}

Poultry is now-a-days one of the most important enterprises of Bangladesh which is growing rapidly in both government and private sectors in order to meet up the constant demand for meat, egg and income generation (DLS 2008). Several studies evaluated production performance of indigenous chicken under semi-scavenging system (Islam 2006) and exotic chicken under intensive but confined management (Khan et al. 2006) whereas Sarker (2006) presented a comparative account of the egg quality of different poultry species in the country. Nahar et al. (2007) performed a comparative evaluation of egg quality and economic importance of the broiler parental stock along with the productivity and profitability of commercial broiler cockerel layer strain and a crossbred Sonali. Subsequently Islam and Nahar (2008) studied some quantitative characteristics of White Leghorn (WLH), Rhode Island Red (RIR) and indigenous breeds. Unfortunately, there is no official estimate of the productivity of poultry farms in the country. There are only about 52,000 registered farms although there exists some 0.3 million farms in the country (Islam 2008), which support overall 1.6\% of the GDP (Parvez 2008). As an important sub-sector of livestock production, however, the poultry plays a crucial role in economic growth that creates numerous employment opportunities (Das et al. 2008).

Billah (2008) reported that 4.5 to 5 metric tons of processed broiler chickens are needed as meat per day in Dhaka City, where about 0.175-0.2 million chickens are consumed per day. Recently Dutta (2010) studied the productivity and management practices of the poultry breeds in Rajshahi City Corporation areas. According to the latest estimate by the Bangladesh Poultry Industries Association (BPIA) and the National Council for Protecting Poultry Farms (NCPPF), however, the number of poultry farms up to June 2009 was 1,14,763, which gradually declined to 97,580 in December 2010 which came further down to around 72,580 in March 2011; and people associated with the poultry enterprise were approximately 50,000,00 (Gupta and Jewel 2011).

Poultry productivity is largely dependent on a number of non-genetic factors such as climate (Elijah and Adedapo 2006), management system and feed supply (Hassen et al. 2006; FSRS 2011), adequate funding (Berning et al. 2008; Das et al. 2008) and seasonal disease outbreaks (Alam et al. 2009; Mengesha et al. 2011; Natukunda 
Saiful et al.

et al. 2011a). On the other hand, studies designed to estimate overall return on investment revealed that incidence of diseases (Ament et al. 1993; Vaillancourt 2001), bio-security measures (Davidson et al. 1999), efficient management of resources (Nworgu and Egbunike 2000), feed and adoption of more innovation (Alabi and Aruna 2005), and growth rate and low productivity (Nworgu 2007; Natukunda et al. 2011b) affect the cost-benefit ratio of poultry farms. In this study, productivity of some selected government and private poultry farms along with their cost-benefit analysis, market prices of the available chicken breeds and constraints of poultry farming in northern districts of the country have been presented.

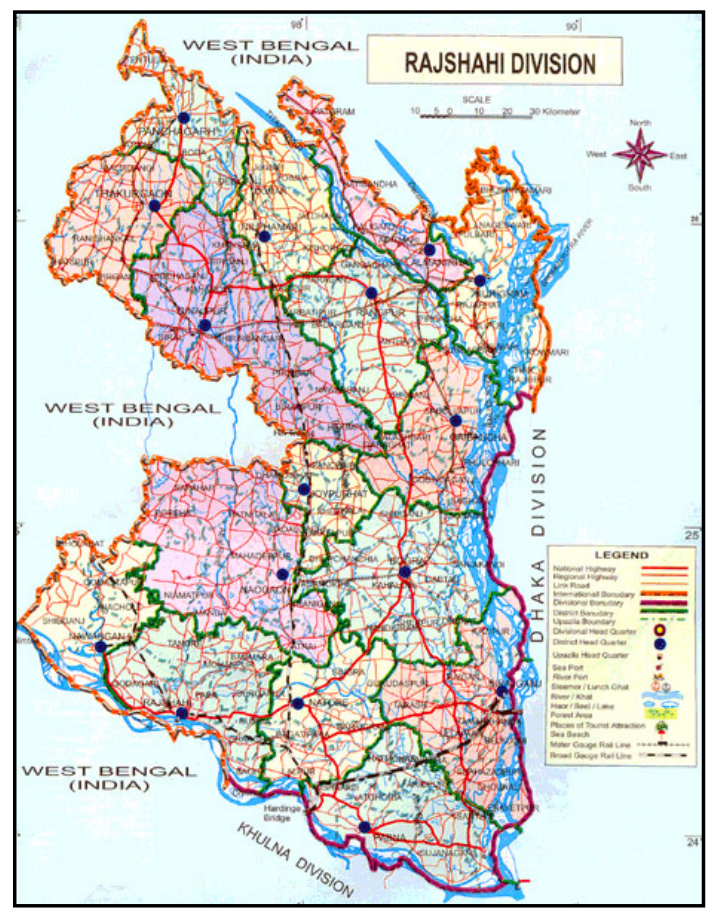

\section{Materials and Methods}

Study area: Seven Districts of the Northern region of Bangladesh under Rajshahi Division, namely Thakurgaon, Dinajpur, Nilphamari, Saidpur, Rangpur, Bogra and Rajshahi, were selected for the study (Plates 1 and 2). The main considerations in selecting the study area were as follows: (a) a large number of poultry farms are raised in the region; (b) no study of this nature was conducted previously; (c) the study areas are well-communicated; (d) satisfactory co-operation received from the farm owners; and (e) occasional outbreaks of bird flu (avian influenza, AI) and other poultry diseases in the area.

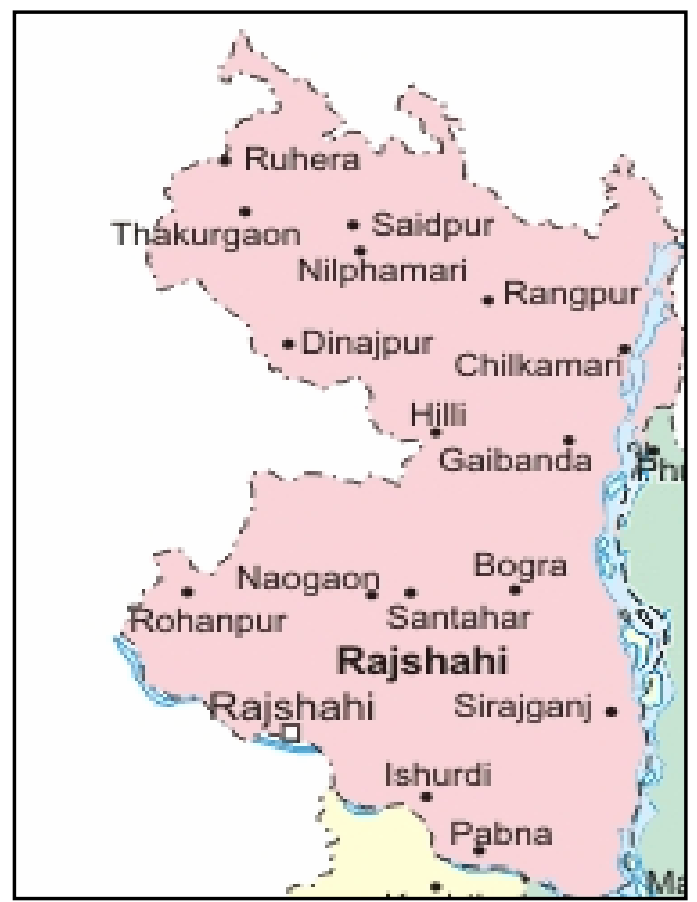

Plates 1-2. Map of Rajshahi Division that includes the study area (left); Map showing Northern Districts under study (right)

Poultry farms: A total of ten poultry farms, consisting of four governments and six private ones, were selected for collecting data and information required for the experiment. The poultry farm owners were interviewed personally and data were collected using a specifically designed interview schedule. The main consideration for selecting the farms was the availability of both pure and crossbred chickens throughout the year. To honour the privacy of the farms, anonymity was adopted by assigning codes $G_{1}$ to $G_{4}$ for the government and $P_{1}$ to $\mathrm{P}_{6}$ for the private farms.

Chicken breeds: Information on productivity, cost-benefit analysis, constraints on mass producing and market prices of five chicken breeds viz. Cobb-500, commonly known as broiler, Fayoumi, ISA Brown, Rhode Island Red (RIR) and Sonali (a hybrid from Fayoumi $+\times \mathrm{RIR}^{\lambda}$ ) were collected from the selected poultry farms.

Parameters studied: The following parameters were studied.

(a) Productivity: Farm- and breed-wise productivity of the chickens in all 10 poultry farms was recorded during the study. Data were collected fortnightly from October 2010 to March 2011.

(b) Cost-benefit analyses: For the cost-benefit analyses (in Tk.) of the farms, the following parameters were computed (Alabi and Aruna 2005; Nworgu 2007): Total cost of production $(\mathrm{TCP})=$ amount spent for rent of the 
land/houses, purchase of day-old chicks, feed, medicine and wages, electricity and water bills, caring and depreciation; Total revenue $(\mathrm{TR})=$ amount received from sales of chicken, eggs and poultry waste products; Net profit $(\mathrm{NP})=$ TR-TCP; Cost-benefit ratio $(\mathrm{CBR})=$ $\mathrm{TR} \div \mathrm{TCP}$; Rate of return on investment $(\mathrm{RRI}) \%=$ $\mathrm{NP} \div \mathrm{TCP} \times 100$; Gross ratio $(\mathrm{GR})=\mathrm{TCP} \div \mathrm{TR}$; and Profitability index $(\mathrm{PI})=\mathrm{NP} \div \mathrm{TR}$. In addition, the production costs and profits/losses per chick, along with the whole-sale and retail-sale prices for each breed were also calculated.

(c) Market prices of the chicken breeds: To get an overall picture of the poultry enterprise and its dynamics on the economy of the study area, the wholesale as well as the retail-sale prices of the chicken breeds were monitored, recorded and analyzed for six months during the study period.

(d) Major constraints of the poultry productivity: In order to recognize the major problems frequently faced by the poultry farm owners, two persons, preferably the Manager and his assistant, or else the next responsible persons, from each farm were asked to pass their comments and/or suggestions on 10 questionnaires involving the capital invested for farming, availability and prices of day-old chick, feed and vaccines, housing systems, power (electricity) supplies, outbreak of poultry diseases including bird flu, marketing and selling systems, credit/loan facilities and training on poultry farming. The queries and their answers reflected the overall constraints and bottlenecks of the poultry enterprise in the study area.

Statistical analyses: Data and various information on productive performance and cost-benefit of different breeds of chickens at the government and private farms were collected and soon afterwards were up-loaded on to a PC for further processing. Moreover, costs of rearing the chicken breeds and their corresponding losses or profits, and the current market prices of the chickens were analyzed. Data on constraints of the poultry productivity were expressed in percentages. One-way analysis of variance (ANOVA) for the productivity data among treatment means was performed. Microsoft Excel spread sheets and SPSS for Windows (version 11.0) were used for analyzing the experimental data.

\section{Results and Discussion}

Productivity: Farm-wise and breed-wise productivity of 10 poultry farms in the study area (Table 1; Fig. 1) reveal that significant differences existed among the poultry farms $\left(\mathrm{F}_{9,103}=29.32 ; \mathrm{P}<0.001\right)$, among the chicken breeds $\left(\mathrm{F}_{4}, 108=3.03 ; \mathrm{P}<0.05\right)$, as well as between the government and private farms $\left(F_{1,111}=\right.$
$6.17 ; \mathrm{P}<0.05)$. A total of $6,41,004$ chickens were produced by the farms under study, of which Cobb-500 grown in P1 was the highest in number $(2,43,000)$ while G1 yielded the lowest, 10,117 heads comprising Fayoumi, RIR and Sonali breeds. The most common chicken breed reared was Cobb-500 (2,76,350) and the least common was ISA Brown $(43,163)$.

Table 1. Farm- and breed-wise productivity of chickens in the poultry farms of the Northern Districts of Bangladesh during October 2010 and March 2011.

\begin{tabular}{cccccccc|}
\hline Farms & Cobb-500 & Fayoumi & $\begin{array}{c}\text { Breeds } \\
\text { ISA Brown }\end{array}$ & RIR & Sonali \\
\hline G1 & 0 & 3374 & 0 & 3371 & 3372 \\
G2 & 0 & 25960 & 0 & 25957 & 25957 \\
G3 & 0 & 14500 & 0 & 14497 & 14503 \\
G4 & 0 & 11552 & 0 & 11547 & 11551 \\
P1 & 243000 & 0 & 0 & 0 & 0 \\
P2 & 2000 & 0 & 20183 & 0 & 0 \\
P3 & 12300 & 0 & 0 & 0 & 0 \\
P4 & 0 & 45433 & 0 & 45433 & 45434 \\
P5 & 0 & 0 & 22980 & 0 & 0 \\
P6 & 19050 & 0 & 0 & 0 & 19050 \\
Breed total & 276350 & 100819 & 43163 & 100805 & 119867 \\
\hline
\end{tabular}

*G1-G4 refer to government and P1-P6 refer to private farms.

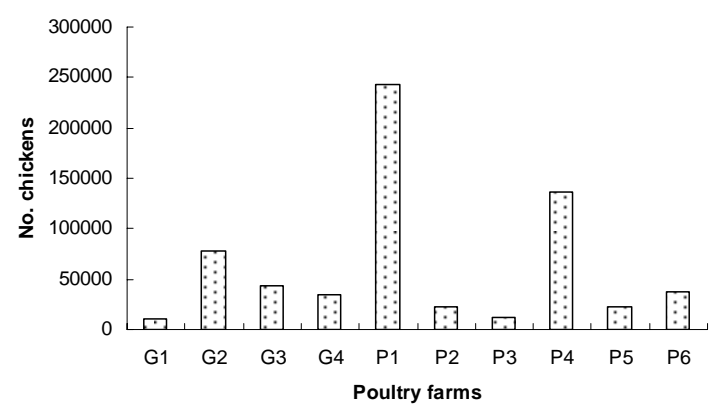

Fig. 1. Productivity of the poultry farms in the northern districts of Bangladesh; G1-G4 refer to government and P1-P6 refer to private farms.

Cost-benefit: A summarized account of the cost-benefit components of the poultry farms is presented in Table 2 . Results clearly demonstrate that all the government farms in the study area were losing concerns in the order of G2 > $\mathrm{G} 3=\mathrm{G} 4>\mathrm{G} 1$, as indicated by their negative NP and RRI values, whereas all the private farms were making profits in the sequence of $\mathrm{P} 1>\mathrm{P} 3>\mathrm{P} 2>\mathrm{P} 6>\mathrm{P} 4=\mathrm{P} 5$ at varying $\mathrm{NP}$ and RRI. This trend has been reflected in terms of the PI values (Fig. 2), which in the government farms ranged between -0.09 and -0.13 , and in the private farms between 0.18 and 0.52 . Other components of the analyses such as CBR and GR also show similar nature of profitability against the farms under study. 
Table 2. Various components of the cost-benefit analysis derived from the total cost of production (TCP) and total revenue (TR) in the poultry farms of the northern districts of Bangladesh during October 2010 and March 2011.

\begin{tabular}{ccccccccc|c|}
\hline Farms & TCP & TR & NP & CBR & RRI & GR & PI \\
\hline G1 & 11109136 & 10217100 & -892036 & 0.92 & -8.03 & 1.09 & -0.09 \\
G2 & 50136254 & 44531650 & -5604604 & 0.89 & -11.18 & 1.13 & -0.13 \\
G3 & 22649848 & 20489875 & -2159973 & 0.90 & -9.54 & 1.11 & -0.11 \\
G4 & 22775040 & 20506025 & -2269015 & 0.90 & -9.96 & 1.11 & -0.11 \\
P1 & 182385840 & 379080888 & 196695048 & 2.08 & 107.85 & 0.48 & 0.52 \\
P2 & 14102096 & 20810925 & 6708829 & 1.48 & 47.57 & 0.68 & 0.32 \\
P3 & 263889 & 418500 & 154611 & 1.59 & 58.59 & 0.63 & 0.37 \\
P4 & 32411850 & 39619375 & 7207525 & 1.22 & 22.24 & 0.82 & 0.18 \\
P5 & 10293136 & 12577150 & 2284014 & 1.22 & 22.19 & 0.82 & 0.18 \\
P6 & 3740773 & 5269500 & 1528727 & 1.41 & 40.87 & 0.71 & 0.29 \\
\hline
\end{tabular}

$\mathrm{RP}=$ total cost of production; TR-total revenue;

$\mathrm{NP}=$ net profit; $\mathrm{CBR}=$ cost-benefit ratio; $\mathrm{RRI}=$ rate of return on investment; $\mathrm{GR}=$ gross ratio; $\mathrm{PI}=$ profitability index.

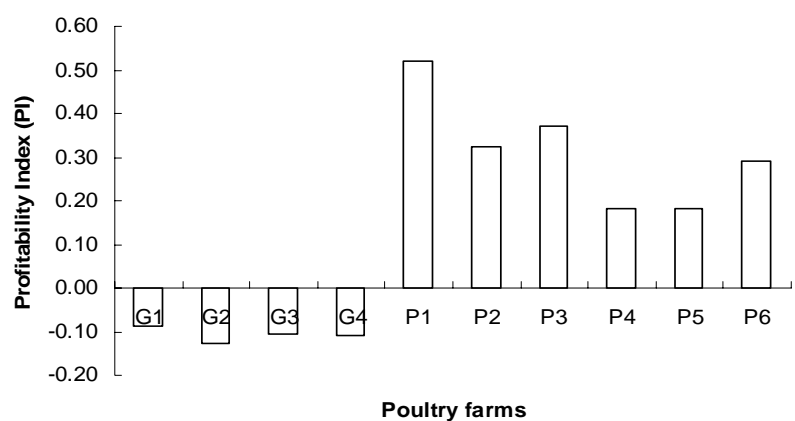

Figure 2 Profitability index (PI) of the of the poultry farms in the Northern Districts of Bangladesh; G1-G4 refer to government and P1-P6 refer to private farms.

Market prices: Depending on the availability of breeds, their supply and demand in the market, the prices of chickens fluctuated throughout the study period (Table 3 ). The average retail-sale price (per $\mathrm{kg}$ ) of the most common breed Cobb 500 was around Tk.109, followed by the least common ISA Brown at Tk. 169, Fayoumi at Tk. 170 and the most popular Sonali at around Tk. 90. The corresponding whole-sale prices of the chicken breeds ranged only between Tk. 5 and Tk.10. In respect of the production cost, however, the maximum profit per chicken was recorded for Fayoumi (Tk. 34), followed by ISA Brown (Tk. 32), Sonali (Tk.14) and Cobb 500 (Tk. 8). Therefore, Cobb 500 was the commonest but the least profitable, and Sonali was the most popular and cheapest one; and the poultry growers achieved the maximum profit from Fayoumi.

Table 3 Breed-wise production, production cost, whole-sale and retail-sale prices, and profit/loss of chickens in the study area during October 2010 and March 2011.

\begin{tabular}{c|c|c|c|c|c|}
\hline $\begin{array}{c}\text { Chicken } \\
\text { breeds* }\end{array}$ & $\begin{array}{c}\text { No. birds } \\
\text { produced }\end{array}$ & $\begin{array}{c}\text { Production cost } \\
\text { per kg (Tk.) }\end{array}$ & $\begin{array}{c}\text { Whole-sale price } \\
\text { per kg (Tk.) }\end{array}$ & $\begin{array}{c}\text { Retail-sale price } \\
\text { per kg (Tk.) }\end{array}$ & $\begin{array}{c}\text { Profit/loss } \\
\text { per kg (Tk.) }\end{array}$ \\
\hline Cobb 500 & 276350 & $103.50 \pm 3.91$ & $104.50 \pm 0.81$ & $109.30 \pm 4.15$ & $7.90 \pm 1.70$ \\
Fayoumi & 100819 & $131.50 \pm 1.12$ & $164.90 \pm 0.83$ & $169.50 \pm 3.50$ & $34.40 \pm 1.80$ \\
ISA Brown & 43163 & $134.80 \pm 0.98$ & $165.10 \pm 0.83$ & $169.00 \pm 3.74$ & $31.50 \pm 1.75$ \\
Sonali & 119867 & $72.60 \pm 0.66$ & $84.80 \pm 0.75$ & $89.50 \pm 3.50$ & $14.40 \pm 1.69$ \\
\hline
\end{tabular}

*RIR was not available in the market during the study period

Major constraints: Most of the respondents (85\%) opined that high feed cost was the most serious concern for keeping the productivity at the demand levels.
Further constraints to maintaining profitable poultry enterprise were recognized to be outbreak of diseases $(80 \%)$, inadequate vaccine and medicine supply at the 
time of need (80\%), lack of easy credit facilities (80\%) and lack of sufficient capital for running the farms $(80 \%)$. However, poor housing for the poultry birds $(75 \%)$, dearth of quality day-old chicks $(70 \%)$, lack of training facilities $(50 \%)$, unpredictable marketing system $(50 \%)$ and assurance for uninterrupted power supply $(40 \%)$ were traced out to be the minor constraints to poultry growers in the study area.

There is a dearth of information regarding the exact productivity in different poultry farms of the country. An early study showed that the location of poultry farms in different agro-ecological zones of the country affected productivity (Rahman et al.1997), who also demonstrated that Sonali had the highest egg production, lowest mortality and high profit per hen. A trial was conducted with different exotic hens reared in semi-scavenging conditions where Sonali was shown to be the best performer crossbred in terms of its higher egg production and better adaptability in rural conditions (Ahmed 1997). Experiments at the Government Central Poultry Farm, Dhaka, during the period from 1987 to 1989 encompassed breeding of 22 genetic groups of five purebreds viz. Australorp, Barred Plymouth Rock, White Leghorn, RIR and Fayoumi, while the remainders were crossbreds $\left(F_{1}\right)$ made up of the aforesaid purebreds (Amber et al. 1999). A later study by Sarkar (2007) showed that Sonali was suitable for the environment of the country. In contrast, Islam and Nahar (2008) reported the productivity of RIR, WLH and indigenous chickens in terms of meat and egg morphometrics where the RIR was assigned to be the superior breed. However, the commercial poultry production in Bangladesh grew tremendously in spite of some difficulties such as bird flu and shortage of day-old chicks, but it made a significant contribution to the economic development of the country (Das et al. 2008). A review made by Faruque et al. (2009) emphasized further research on the genetics, breeding and productivity of poultry species in the country. However, Lemlem and Tesfay (2010) studied productivity of Fayoumi, RIR and WLH and concluded that RIR and WLH can be effectively managed for meat and egg production under scavenging condition by smallholder farmers.

Since the first ever diagnosis of AI in the country on 15 March 2007, the poultry enterprise in Bangladesh has suffered an enormous loss of Tk. 127.5 million during 2007-08, when 745 poultry farms were shut down, 22,47,282 chickens were culled and 30,31,395 eggs were destroyed (DLS, 2008; Alam et al., 2009; Gupta and Jewel, 2011; WHO, 2011). Amidst such an alarming situation, the present investigation was undertaken to evaluate production performance of the selected poultry farms where G2 was attacked by fowl pox, G4 by E. coli infection and salmenellosis, whereas P1 and P2 farms were struck by bird flu, P3 by gumboro and omphalitis, P5 by common cold and P6 by gumboro, Newcastle and hypervitaminosis. Irregular incidences of these diseases therefore had obvious effects on the productivity of the poultry farms under study. In agreement with some earlier findings where medication and vaccination provided respectively $32 \%$ and $76 \%$ protection against Newcastle and gumboro diseases (Aganga et al. 2000) and vaccinated birds showed higher antibody against Newcastle disease (Schmidt et al. 2010), vaccination schedules at G1, G3 and P4 farms provided a total protection against diseases in their flocks.

The cost-benefit assessment of a poultry enterprise is often determined by the level of risk to which the reared flocks are exposed to bio-security measures (Ament et al. 1993; Davidson et al. 1999; Vaillancourt 2001). Apart from these, the profit margin in poultry production depends mainly on feed utilization, cost of day-old chicks and efficient management of such resources as land, day labourers and appliances (Nworgu and Egbunike 2000). Studies on the financial dynamics of smallholder farms, poultry production and profits can be increased by the use of appropriate feed, capital, vaccines and adoption innovative approaches (Alabi and Aruna 2005; Nahamya et al. 2006; Nworgu 2007). The present losses incurred by the government farms lend support to the work of Kryger et al. (2005), where model-breeders and mini hatcheries were delivering economic losses to the farmers in the DANIDA-supported smallholder poultry-production projects in Bangladesh. In contrast, Berning et al. (2008) estimated rate of return from poultry farms in the urban and semi-urban communities in Barisal City to be 0.42 which is close to $0.32-0.52$ PI values for some private farms of our study, whereas the costbenefit ratios for Fayoumi (5.74) and Sonali (5.92) reported by Zaman et al. (2008) appeared to be much higher than those of the present results. However, finding by Natukunda et al. (2011b) in Uganda seems to be similar to ours where the profit of the poultry farms was affected by total average costs, distance to the nearest market, access to extension services and education level and experiences of the farm owners.

Owing to the unavailability of literature on market prices of various chicken breeds in the country, the present prices cannot be compared. But it has clearly been shown that Cobb 500 was the cheapest breed, followed by ISA Brown, Fayoumi and Sonali while RIR was not being sold in the markets during the study period. 
Feed stuff substitution (Rae and Hartel 1998), housing (Elijah and Adedapo 2006), government funding (Das et al. 2008), mortality by infectious diseases (Permin and Madsen 2002; Sonaiya and Swan 2004; Natukunda et al. 2011a; Mengesha et al. 2011) and feed ingredient market in SE Asia (FSRS 2011) were recognized to be the usual constraints for poultry raising. In partial agreement with the present results, Sonaiya and Swan (2004) pointed out that small credit, supplying parent stocks and supplementary feeds, participatory training, delivery of vaccines, day-old chickens, training and follow-up are the major problems for poultry farmers. Elijah and Adedapo (2006) showed that in order to improve poultry productivity, the poultry farmers need to establish appropriate housing and sanitation practices to minimize the effect of heat and the occurrence and spread of diseases. Seasonal disease outbreaks (mainly Newcastle disease), predation, low productive performance of certain breeds, theft, inadequate chicken management and lack of chicken marketing information were identified as the most important constraints affecting the chicken enterprise. Top five constraints recognized in this study viz. high feed cost, outbreak of diseases, inadequate vaccine/medicine supplies, insufficient credit facilities and lack of capital conform to the factors emphasized by Sonaiya and Swan (2004) and Das et al. (2008), FSRS (2011) and Mengesha et al. (2011).

This study appears to be the first one in connection with the estimation of productivity and cost-benefit analyses of some selected poultry farms of the northern Districts of Bangladesh. Significant differences were found among the farms, chicken breeds and between the government and private farms. The most common breed was Cobb 500 whereas the least common was ISA Brown. Interestingly all the government farms were running in loss compared to their private counterparts as indicated by the profitability indices. The commonest but the least profitable breed was Cobb 500, but the most popular and cheapest breed was Sonali, and the growers achieved the maximum profit from Fayoumi. High feed cost, outbreak of diseases, inadequate supply of vaccines/ medicines, lack of credit facilities and capital funding were recognized to be the major constraints. It finally appeared from the above findings that strict bio-safety oriented management practices coupled with the selection of fast growing and heavy laying breeds of chickens could ensure profitability of the poultry farms in the study area.

Acknowledgements: This forms a part of doctoral work by MAK, who is grateful to the Chairman, Department of Zoology, Rajshahi University, for providing necessary facilities. The co-operation and assistance received from the farm managerial bodies and poultry owners are also thankfully acknowledged. The work was partly supported by a research grant to MS from the University Grants Commission (UGC) of Bangladesh through Rajshahi University.

\section{References}

Aganga AA, Omphile UJ, Malope P, Chabanga CH, Motsamai GM \& Motsumi LG. 2000. Traditional poultry production and commercial broiler alternatives for small-holder farmers in Botswana. Livestock Res. Rural Dev. 12(4): http://www.lrrd.org/ lrrd12/4/cont1204.htm.

Ahmed Z. 1997. Semi-scavenging poultry model production chin. Impact of smallholder livestock development project in some selected areas of Bangladesh. Livestock Res. Rural Dev. 9(4): http://www.lrrd.org/ lrrd09/4/cont0904.htm.

Alabi RA \& Aruna MB. 2005. Techinical efficiency of family poultry production in Niger-delta, Nigeria. J. Cent . Eur. Agric. 6(4): 531-538.

Alam J, Giasuddin M, Samad MA \& Taimur MJFA. 2009. Avian influenza: Review on recent evidence of Bangladesh. J. Asiat. Soc. Bangladesh, Sci. 35(1): 87-100.

Amber MAJ, Bhuiyan AKFH, Hoque MA \& Amin MR. 1999. Ranking of some pure and crossbred chicken using scoring indices. Indian J Poultry Sci 34(2): 140-146.

Ament AJ, Jansen J, van de Giessen A \& Notermans S. 1993. Costbenefit analysis of a screening strategy for Salmonella enteritidis in poultry. Vet. Quart. 15(1), 33-37.

Berning C, Correa B, Sirman K \& Sosa F. 2008. Homestead food production in Barisal, Bangladesh. International Development Studies, Elliot School of International Affairs, George Washington Univ., USA. 72 pp.

Billah M. 2008. Bird flu and our media. Daily Star 43, 26 February.

Das SC, Chowdhury SD, Khatun MA, Nishibori M, Isobe N \& Yoshimura Y. 2008. Poultry production profile and expected future projection in Bangladesh. World's Poultry Sci. J. 64: 99-118.

Davidson S, Gallipan D, Eckert TE, Ziegler AF \& Eckroade RJ. 1999. Economic analysis of an outbreak of avian influenza, 1997-1998. J. Am. Vet. Med. Assoc. 214(8): 1164-1167.

DLS (Directorate of Livestock Services) 2008. Livestock Statistics. Memeogram. Dhaka, Bangladesh.

Dutta RK. 2010. Body and egg morphometrics and haematobiochemical values of some chicken breeds (Gallus domesticus L.) with their management practices and production performance. MSc thesis, Dept. of Zool. Rajshahi Univ. $210+37$ pp.

Elijah OA \& Adedapo A. 2006. The effect of climate on poultry productivity in Ilorin Kwara State, Nigeria. Int J Poultry Sci. 5(11): 1061-1068.

Faruque MO, Khan MYA \& Asaduzzaman M. 2009. Poultry genetics and breeding in Bangladesh: past, present and future. $6^{\text {th }}$ International Poultry Show and Seminar, WPSA-BB, pp.163-167.

FSRS (Frost and Sullivan Research Service) 2011. Analysis of the Southeast Asian feed ingredients market. Frost and Sullivan Sales Dept., Asia Pacific Region, Singapore. Published 3 October 2011.

Gupta TD \& Jewel SI. 2011. Poultry destruction by 'bio-conspiracy' (in Bangla). The Daily Kaler Kantha. 27 March 2011.

Hassen H, Neser FWC, Dessie T, de Kock A \& Marle-Koster EV. 2006. Studies on the growth performance of native chicken ecotypes and RIR chicken under improved management system in Northwest Ethiopia. Livestock Res. Rural Dev. 18(6): http://www.lrrd.org/ lrrd18/06/cont1806.htm.

Islam MA. 2006. Comparative egg production and egg quality of Indigenous full feathered and naked neck chicken at hot-humid climate. Bang. J. Anim. Sci. 35(1-2): 99-105. 
Islam MN 2008. To protect the bird flu the help of Bangladesh Arms force is being taken, all necessary steps from every corner (in Bangla) Daily Ittefaq 55(88): 25 March.

Islam MS \& Nahar MS. 2008. An analysis of some quantitative characters of the white Leghorn, Rhode Island Red and Local chicken breeds. $16^{\text {th }}$ Biennial Int. Conf. Zool. Soc. p.70.

Kryger KN, Riise JC, Sarker PK, Mustafa G \& Bell JG. 2005. From a model to a learning approach: The impact of DANIDAsupported smallholder poultry-production projects in Bangladesh. Proc. Int. Conf. held in Der es Salaam, Tanzania, 5-7 October, 2005 (eds. Alders RG, Spradbrow PB and Young MP). p. 51.

Lemlem A \& Tesfay Y. 2010. Performance of exotic and indigenous poultry breeds managed by smallholder farmers in northern Ethiopia. Livestock Res. Rural Dev. 22(7): http://www.lrrd.org/ lrrd22/7/cont2207.htm.

Mengesha M, Tamir B \& Dessie T. 2011. Village chicken constraints and traditional management practices in Jamma District, South Wollo, Ethiopia. Livestock Res. Rural Dev. 23(2): http://www.lrrd.org/ lrrd23/2/cont2302.htm.

Nahamya FH, Mukiibi-Muka G, Nasinyama GW \& Kabasa JD. 2006. Assessment of the cost effectiveness of vaccinating free range poultry against Newcastle disease in Busedde sub-county, Jinja District, Uganda. Livestock Res. Rural Dev. 18(11): http://www.lrrd.org/ lrrd18/11/cont1811.htm.

Nahar K, Ahmed S, Monir MM \& Rahman MS. 2007. Comparative evaluation of egg quality characteristics of broiler parent stock and synthetic broiler. Bang. J. Anim. Sci. 36(1-2): 82-87.

Natukunda K, Kugonza DR \& Kyarisiima CC. 2011a. Indigenous chickens of the Kamuli Plains in Uganda: I. Production system and flock dynamics. Livestock Res. Rural Dev. 23(10): http://www.lrrd.org/ lrrd23/10/cont2310.htm.

Natukunda K, Kugonza DR \& Kyarisiima CC. 2011b. Indigenous chickens of the Kamuli Plains in Uganda: II. Factors affecting their marketing and profitability. Livestock Res. Rural Dev. 23(10): http://www.lrrd.org/ lrrd23/10/cont2310.htm.

Nworgu FC. 2007. Economic importance and growth rate of broiler chickens served fluted pumpkin (Telfaria occidentalis) leaves extract. Afr. J. Biotechnol. 6(2): 167-174.

Nworgu FC \& Egbunike GN. 2000. Performance and nitrogen utilization of broiler chicks fed full fat extruded soybean meal and full fat soybean. Trop. Anim. Prod. Invest. J. 3: 47-54.
Parvez S. 2008. Poultry farmers' livelihood at stake in the face of bird flu. Daily Star XVIII (20): 3 February.

Permin A \& Madsen M. 2002. Literature review on disease occurrence and impact (smallholder poultry). In: Investing in Animal Health Research to Alleviate Poverty (eds. Perry BD, Randolf TF, McDermott JJ, Sones KR and Thornton PK). pp. 1-6. International Livestock Research Institute, Nairobi Kenya.

Rae AN \& Hartel TW. 1998. Livestock productivity convergence in the Asia-Pacific Region: Impacts on trade in livestock products and grains. Ann. Conf. New Zealand Assoc. Econ. Wellington, 2-4 September 1998. 1-24 pp.

Rahman M, Sorensen P, Jensen HA \& Dolberg F. 1997. Exotic hens under semi scavenging conditions in Bangladesh. Livestock Res. Rural Dev. 9(3): http://www.cipav.org.co/lrrd/ lrrd9/3/bang931.htm.

Sarkar PK. 2007. Comparative study on the productivity and profitability of commercial broiler cockerel of a layer strain and crossbreed (RIR $\hat{\sigma} \times$ Fayoumi + ). MS thesis, Dept. of Poultry Science, Bangladesh Agricultural University, Mymensingh, 32 pp.

Sarker SI. 2006. A comparative study on egg quality of few breeds of different poultry species. MS thesis, Dept. of Poultry Science, Bangladesh Agricultural University, Mymensingh, 128+14 pp.

Schmidt EMS, Paulillo AC, Martins GRV, Denadai J. \& Lapera IM. 2010. Immunological and clinical parameters of Newcastle disease vaccination in Bronze Turkeys (Meleagris gallopavo). Int. J. Poultry Sci. 9(2): 180-182.

Sonaiya, EB \& Swan SEJ. 2004. Small-scale Poultry Production: Technical Guide. FAO Annual Production and Health. Chapter 8: Production Economics. 1. Food and Agriculture Organization of the United Nations, Rome. 125 pp.

Vaillancourt JP. 2001. How do you determine the cost-benefit of a biosecurity system? Dept. of Farm Animal Health and Resource Management, North Carolina State Univ., USA. 10 pp.

WHO (World Health Organization) 2011. Antigenic and genetic characteristics of influenza A (H5N1) and influenza A (H9N2) viruses for the development of candidate vaccine viruses for pandemic preparedness. WHO Newsletter on H5-H9 Vaccine Virus Update, February 2011.

Zaman MA, Sorensen P, Karim MR \& Rume FI. 2008. Study on the laying rate and cost-benefit ratio of semi-scavenging hens fed with different levels of supplementary feed. Bangladesh Res. Pub. J. 1(1): 38-36.

Manuscript received on 19 May 2012 and revised on 20 October 2012 